\title{
Low-frequency linear waves and instabilities in uniform and stratified plasmas: the role of kinetic effects
}

\author{
K. M. Ferrière \\ Observatoire Midi-Pyrénées, Toulouse, France
}

Received: 13 September 2004 - Revised: 23 November 2004 - Accepted: 24 November 2004 - Published: 23 December 2004

Part of Special Issue "Advances in space environment turbulence"

\begin{abstract}
We review the basic approximations underlying magnetohydrodynamic (MHD) theory, with special emphasis on the closure approximations, i.e. the approximations used in any fluid approach to close the hierarchy of moment equations. We then present the main closure models that have been constructed for collisionless plasmas in the large-scale regime, and we describe our own mixed MHD-kinetic model, which is designed to study low-frequency linear waves and instabilities in collisionless plasmas. We write down the full dispersion relation in a new, general form, which gathers all the specific features of our MHD-kinetic model into four polytropic indices, and which can be applied to standard adiabatic MHD and to double-adiabatic MHD through a simple change in the expressions of the polytropic indices. We study the mode solutions and the stability properties of the full dispersion relation in each of these three theories, first in the case of a uniform plasma, and then in the case of a stratified plasma. In both cases, we show how the results are affected by the collisionless nature of the plasma.
\end{abstract}

\section{Introduction}

Magnetohydrodynamic (MHD) theory constitutes a convenient tool to study the behavior of magnetized plasmas on temporal and spatial scales much larger than the scales (gyroperiod and Larmor radius, respectively) characterizing the gyromotion of individual particles, and subject to the requirement that all the characteristic velocities (Alfvén speed, sound speed, bulk velocity of the plasma, and phase speed of the waves) be much less than the speed of light. In general, it is also assumed that the different (ion and electron) populations composing the plasma maintain a state of quasi-perfect charge neutrality, for instance through collisions (when the timescales involved are much longer than the ion-electron collision time) or through electric coupling (when the length-

Correspondence to: K. M. Ferrière

(ferriere@ast.obs-mip.fr) scales involved are much greater than the Debye length). The plasma is then treated as a single fluid satisfying the three standard fluid (mass, momentum, and thermal pressure) equations together with Maxwell's equations.

The MHD mass and momentum equations are obtained by summing the namesake equations of the individual plasma components, which, in turn, correspond exactly to the zerothand first-order moments, respectively, of the kinetic (Boltzmann or Vlasov) equation. In this sense, the individual mass and momentum equations are exact. The MHD mass equation, which does not rely on the basic MHD assumptions (large time/length scales, low velocities, quasi-neutrality), is also exact. However, the MHD momentum equation is not strictly exact, insofar as the MHD assumptions of low velocities and quasi-neutrality are explicitly used to neglect the electric force.

Formally, the global pressure equation is obtained by summing the individual pressure equations, which correspond to the second-order moment of the kinetic equation. The exact pressure equations contain several non-adiabatic terms, arising from viscous stresses, heat fluxes, and inter-species collisions (in the individual equations) or Ohmic dissipation (in the global equation). In ideal MHD, the non-adiabatic terms are neglected, with the result that the pressure equations reduce to the standard adiabatic equation of state. Neglect of the non-adiabatic terms in the individual pressure equations is justified when the transport coefficients (viscosity and thermal conductivity) are very small (generally true for very high self-collision frequencies, given that the transport coefficients are proportional to the self-collision time) and when the inter-species collision term goes to zero (true not only in the limit of very low inter-species collision frequencies, but also in the opposite limit of very high inter-species collision frequencies, in which inter-species drifts become vanishingly small). More importantly here, in the global pressure equation, the adiabatic approximation is justified for very small transport coefficients and negligible Ohmic dissipation, both of which are generally achieved in very highly collisional plasmas - the former for the usual reason reminded above 
and the latter because very high ion-electron collision frequencies entail very weak electric current densities. Note that for very high inter-species collision frequencies, the global adiabatic pressure equation can be directly deduced from the individual adiabatic pressure equations, because in this case the bulk velocities of the plasma components that contribute to the thermal pressure differ only very little from the mean bulk velocity, which in turn implies that the total thermal pressure is approximately equal to the sum of the individual pressures.

The set of Maxwell's equations is simplified as well. In Faraday's equation, the electric field is eliminated by means of Ohm's law, which is obtained by subtracting the electron momentum equation from the full ion momentum equation and dropping the terms that become negligible in the MHD limit. This leads to an evolution equation for the magnetic field, customarily referred to as the induction equation. In Ampere's equation, the displacement current is neglected, by virtue of the MHD assumptions of low velocities for the transverse component and quasi-neutrality for the longitudinal component. The ensuing simplified equation constitutes only a secondary equation used to eliminate the electric current density in favor of the magnetic field in the momentum equation and, if necessary, in Ohm's law; it also serves to calculate the current density once the whole MHD problem has been solved.

It is clear that the derivation of the MHD equations involves two levels of approximation. At the first level, a finite set of fluid equations is deduced from the kinetic equation for each plasma component. These fluid equations are obtained by taking the successive moments of the kinetic equation, and the last moment equation is simplified in such a way as to get rid of all higher-order moments and thus close the system. The approximations introduced in the last moment equation (the closure equation) can only be justified on a case-by-case basis, with arguments that often ultimately draw on high collision frequencies. At the second level, a closed set of MHD equations is deduced from the above set of multi-fluid equations combined with Maxwell's equations. The approximations made at this stage are almost entirely justified by the basic MHD assumptions of large time/length scales, low velocities, and quasi-neutrality, although for the pressure equation, one sometimes also has to explicitly invoke very strong inter-species collisionality.

The closure problem is inherent in all fluid descriptions, sophisticated though they may be. The adiabatic assumption constitutes one possible way to close the hierarchy of fluid equations, but various other closure approximations can be found in the literature, all with the objective of having the closure equation capture as well as possible the relevant physics contained in the full kinetic equation. The vast majority of existing closure approximations rely, directly or indirectly, on high collision frequencies. There exist also a number of closure formalisms for weakly collisional or collisionless plasmas, but most of them are either ill-founded or restricted to particular cases (e.g. linear waves). Since in practice space plasmas are often collisionless or only weakly collisional, it is highly desirable to improve the way such plasmas are currently modelled.

The first thing to realize is that in the absence of (frequent) collisions, the plasma thermal pressure has no reason to be isotropic (unless there exist efficient collisionless isotropization mechanisms, such as pitch-angle scattering by high-frequency waves). On the large temporal and spatial scales characteristic of the MHD regime, the small-scale gyromotion of particles ensures pressure isotropy in planes perpendicular to the magnetic field, while leaving the parallel pressure free to evolve independently. As a result, the plasma thermal pressure has two distinct components, perpendicular $\left(P_{\perp}\right)$ and parallel $\left(P_{\|}\right)$, respectively, to the magnetic field, in which case the plasma is said to be gyrotropic. Another important consequence of the small-scale gyromotion of particles is that the off-diagonal elements of the stress tensor, which represent the viscous stresses, are negligible.

Under MHD conditions, a gyrotropic plasma satisfies the same mass and momentum equations as an isotropic collisional plasma, with this difference that the thermal pressure tensor in the momentum equation no longer reduces to the scalar pressure $P$, but instead contains the two distinct components $P_{\perp}$ and $P_{\|}$. Accordingly, the single equation of state for $P$ must be replaced by a pair of equations for $P_{\perp}$ and $P_{\|}$, respectively. The closure problem then consists of finding appropriate equations for $P_{\perp}$ and $P_{\|}$.

The simplest and most-widely used closure approximation is analogous to that leading up to the adiabatic equation of state in isotropic collisional MHD. For each plasma component, the heat-flux terms (the only remaining non-adiabatic terms) are neglected in the second-order moments of the kinetic equation, which then reduce to simple evolution equations for the perpendicular and parallel thermal pressures in terms of density and magnetic field strength, usually referred to as the double-adiabatic equations of state. The plasma components that contribute to the thermal pressures are then assumed to have bulk velocities almost equal to the mean bulk velocity, whereupon the perpendicular and parallel thermal pressures of the whole plasma satisfy the same doubleadiabatic equations of state as the individual pressures.

The double-adiabatic equations were first derived by Chew et al. (1956) in the simple case of a two-component plasma with cold electrons (so that only the ions contribute to the thermal pressures) and no external forces. Chew et al. (1956) were careful to emphasize that the double-adiabatic equations are valid only in very special circumstances. The reason why they fail in general is because in the absence of (frequent) collisions, the heat flux along magnetic field lines may generally not be neglected. Despite this clear warning, the double-adiabatic equations, also known as the CGL equations, are often used without proper judgment to describe the pressure response of gyrotropic plasmas.

There have been attempts to develop CGL-like formalisms for collisionless plasmas, which would preserve the intrinsic simplicity of the double-adiabatic closure, while giving results in better agreement with kinetic theory. For instance, Hau and Sonnerup (1993) proposed a double-polytropic 
MHD model to study low-frequency linear waves in a homogeneous medium. In their model, the equations of state for $P_{\perp}$ and $P_{\|}$have the same form as the double-adiabatic equations, but they are written in terms of two polytropic indices, $\gamma_{\perp}$ and $\gamma_{\|}$, whose values are not specified a priori. The freedom in the choice of the polytropic indices' values makes it possible to better describe the plasma behavior in specific situations. However, no single choice leads to the correct wave properties throughout the low frequency range. In this sense, the double-polytropic approach may not be considered as fundamentally superior to its double-adiabatic counterpart.

The validity of the adiabatic closure in collisionless twocomponent plasmas was discussed by Belmont and Rezeau (1987) in the context of linear waves with frequency below the ion gyrofrequency. They considered a homogeneous background medium, assumed that ions and electrons had isotropic Maxwellian distributions (and, hence, isotropic pressures) at equilibrium, and took first-order finite Larmor radius effects into account. They showed that correct fluidtype closure equations could be obtained for waves with a parallel phase speed either much higher (adiabatic limit) or much lower (isothermal limit) than the thermal speed of the considered species. They also derived, from the perturbed Vlasov equation, a general closure equation giving the correct expression of the pressure tensor perturbation.

Belmont and Mazelle (1992) pursued the work of Belmont and Rezeau (1987), although in a slightly different context. They focused on lower-frequency waves, for which they were entitled to ignore finite Larmor radius effects, but they allowed the equilibrium distribution functions to be anisotropic bi-Maxwellians (leading to gyrotropic pressures). They showed that for any given wave mode, the correct relations for the thermal pressure perturbations (chosen again as closure equations) could be written in the form of polytropic laws, and they derived the correct expressions of the polytropic indices for each mode, starting again from the perturbed Vlasov equation.

The idea of employing the correct equations of state for $P_{\perp}$ and $P_{\|}$and incorporating them into an MHD-like theory has led to various mixed MHD-kinetic models for low-frequency phenomena in collisionless plasmas. Cheng (1991) and later Cheng and Johnson (1999) constructed such hybrid models in general magnetic field geometries including magnetic pressure gradients, field line curvature, and magnetic trapping regions. Cheng (1991) restricted his attention to plasmas consisting of a low-energy component, for which he used the CGL equations, and a rarefied energetic component, for which he used the kinetically correct equations of state. Cheng and Johnson (1999) generalized Cheng's (1991) model by allowing for multiple ion species and treating them all correctly from a kinetic point of view. In this manner, they retained important kinetic effects such as finite Larmor radii, resonant wave-particle interactions, and trapped particle dynamics for all the species. Both papers provide a complete set of integro-differential eigenmode equations for linear waves with frequency less than the ion gyrofrequencies.
Hammett and Perkins (1990) and Snyder et al. (1997) developed other hybrid models, which they referred to as Landau-fluid, because these models contain a closed set of fluid moment equations, which incorporate the kinetic effects associated with linear parallel Landau damping. Whereas Hammett and Perkins (1990) limited themselves to the electrostatic case (no magnetic perturbations) but included finite Larmor radius effects, Snyder et al. (1997) developed fully electromagnetic models but focused on the MHD limit in which finite Larmor radius effects are negligible. For simplicity, the plasma was supposed to contain only electrons and one species of singly-charged ions. The authors worked out two types of models: an elaborate $4+2$ model, which evolves 4 parallel moments $\left(n, v_{\|}, P_{\|}, q_{\|}\right)$and 2 perpendicular moments $\left(P_{\perp}, q_{\perp}\right)$, and a simpler $3+1$ model, which evolves 3 parallel moments $\left(n, v_{\|}, P_{\|}\right)$and 1 perpendicular moment $\left(P_{\perp}\right)$. In both models, the closure was chosen such as to ensure nonlinear conservation of mass, momentum, and energy, and at the same time yield a linear response function that closely matches that predicted by kinetic theory for a bi-Maxwellian plasma.

Chang and Callen (1992a, b) adopted an alternative Landau-fluid approach, which applies not only to collisionless plasmas, but also to plasmas with arbitrary collisionality. Their system of fluid equations contains the first three moment equations of each plasma species, with stress forces and heat fluxes included and with the thermal pressure assumed isotropic. They first derived the nonlinear perturbed version of their fluid equations in a sheared slab geometry and for small but finite Larmor radii. Then they calculated the kinetically exact linear closure equations for the parallel stress force and the parallel heat flux, using a drift-kinetic Chapman-Enskog-like equation. They showed that this closure automatically brings in the desired kinetic effects, such as Landau damping, diamagnetic drift, and finite Larmor radii.

Going back to strictly collisionless plasmas, Passot and Sulem (2003) constructed a Landau-fluid model which, in addition to incorporating linear Landau damping, also retains finite Larmor radius corrections to the gyrotropic pressures and includes the Hall effect as well as the electron pressure gradient in Ohm's law. They, too, made the simplifying assumption that the plasma is composed of electrons and one species of singly-charged ions, both with bi-Maxwellian distributions at equilibrium. As in Snyder et al. (1997) 3+1 model, they closed the hierarchy of fluid equations at the level of the heat fluxes, but they did so in a more refined manner, by extrapolating the asymptotically exact expressions of the heat fluxes given by a reductive perturbative expansion of the Vlasov-Maxwell equations for long parallel Alfvén waves. With this closure, Passot and Sulem's (2003) model yields the same response functions as the more cumbersome 4+2 model of Snyder et al. (1997). Moreover, although it was initially designed to accurately describe the weakly nonlinear dynamics of dispersive long parallel Alfvén waves, it is also able to correctly reproduce the Landau damping of long oblique magnetosonic waves, and it can be used to study 
oblique and kinetic Alfvén waves in the regime of adiabatic protons and isothermal electrons, subject to the additional condition that the Alfvén speed be much higher than the proton thermal speed and much lower than the electron thermal speed (see Passot and Sulem, 2004).

As discussed above, an important advantage of mixed MHD-kinetic models is that they combine the formal simplicity of double-adiabatic MHD with the capability to provide a kinetically correct description of linear waves in the low-frequency limit. Turning this advantage to good account, Ferrière and André $(2002,2003)$ and André and Ferrière (2004) developed a mixed MHD-kinetic formalism to derive the correct and complete dispersion relation of lowfrequency linear waves in gyrotropic collisionless plasmas and to perform a systematic analysis of their stability properties. They successively considered a homogeneous plasma (Ferrière and André, 2002), a stratified two-component ionelectron plasma (Ferrière and André, 2003), and a stratified multi-component plasma (André and Ferrière, 2004), each time with bi-Maxwellian distributions at equilibrium. Although some of the mixed MHD-kinetic models described above included kinetic effects associated with finite Larmor radii, Ferrière and André truly focused on the low-frequency limit corresponding to the MHD regime and they only retained the kinetic corrections required for consistency with kinetic theory, namely, those associated with linear Landau damping.

The object of the present paper is to put Ferrière and André's work in context with other studies of the same kind and to present their main results in a new, unified manner, which brings to light the similarities and differences between the three different formalisms (isotropic-adiabatic MHD, gyrotropic-double-adiabatic MHD, and gyrotropicexact MHD-kinetic theory) and clarifies the respective roles played by collisionality, pressure anisotropy, and stratification in the stability of the plasma. Our analysis, which is strictly linear, constitutes a first step toward a complete understanding of the behavior of low-frequency perturbations, including their possible development into the nonlinear regime.

In Sect. 2, we review the basic equations entering each of the three formalisms. In Sect. 3, we present the full dispersion relation, written in terms of four polytropic indices and four independent parameters governing the anisotropy (firehose and mirror) instabilities, on the one hand, and the stratification or quasi-interchange (interchange and Parker) instabilities, on the other hand. We also provide the values or expressions of the polytropic indices in the three formalisms. In Sect. 4, we discuss the solutions of our dispersion relation in a uniform plasma, starting with the nature of the different modes and their behaviors at perpendicular and parallel wave vectors, and proceeding with their stability properties in the three formalisms. In Sect. 5, we turn our attention to a stratified plasma. We first introduce the concept of quasiinterchange modes, which, by definition, represent all the modes significantly affected by stratification. We then explain how, in the absence of rotation, stratification modifies the stability properties of the different modes in the three formalisms. In Sect. 6, we conclude our study.

\section{Governing equations}

Let us consider an ideal, multi-component plasma embedded in a magnetized, stratified, and rotating system (such as a planetary magnetosphere). The set of equations governing the temporal evolution of this plasma comprises the MHD mass equation,

$\frac{\partial \rho}{\partial t}=-\nabla \cdot(\rho \boldsymbol{V})$,

the MHD momentum equation,

$$
\begin{aligned}
\rho \frac{D \boldsymbol{V}}{D t}= & -\boldsymbol{\nabla} \cdot \mathbf{P}+\frac{1}{\mu_{0}}(\nabla \times \boldsymbol{B}) \times \boldsymbol{B} \\
& +\rho \boldsymbol{g}-2 \rho \boldsymbol{\Omega} \times \boldsymbol{V},
\end{aligned}
$$

the MHD induction equation in the perfectly-conducting limit,

$\frac{\partial \boldsymbol{B}}{\partial t}=\nabla \times(\boldsymbol{V} \times \boldsymbol{B})$,

and the appropriate equation(s) of state (see below). The notation used here is standard: $\rho$ is the mass density of the plasma, $\boldsymbol{V}$ its bulk velocity, $D / D t \equiv \partial / \partial t+\boldsymbol{V} \cdot \boldsymbol{\nabla}$ the Lagrangian derivative, $\mathbf{P}$ the thermal pressure tensor (with contributions from the different plasma components), $\boldsymbol{B}$ the magnetic field, $\boldsymbol{g}$ the effective gravity (including a centrifugal component), $\boldsymbol{\Omega}$ the angular velocity of the system, $P_{\mathrm{M}} \equiv$ $B^{2} /\left(2 \mu_{0}\right)$ the magnetic pressure, $\widehat{\boldsymbol{e}}_{\mathrm{B}}$ the unit vector along $\boldsymbol{B}$, $\boldsymbol{c} \equiv \widehat{\boldsymbol{e}}_{\mathrm{B}} \cdot \nabla \widehat{\boldsymbol{e}}_{\mathrm{B}}$ the magnetic curvature vector, $\widehat{\boldsymbol{e}}_{\mathrm{g}}$ the unit vector along $\boldsymbol{g}$, and $\mathbf{I}$ the unit dyadic. For future reference, let us note that the magnetic force in Eq. (2) can be rewritten as

$\frac{1}{\mu_{0}}(\nabla \times \boldsymbol{B}) \times \boldsymbol{B}=-\nabla_{\perp} P_{\mathrm{M}}+2 P_{\mathrm{M}} \boldsymbol{c}$.

In isotropic collisional MHD, the thermal pressure tensor reduces to a scalar,

$\mathbf{P}=P \mathbf{I}$,

and its divergence reads

$\nabla \cdot \mathbf{P}=\nabla P$.

In the ideal case, the evolution equation for $P$ is simply the standard adiabatic equation of state,

$\frac{D}{D t}\left(\frac{P}{\rho^{\gamma}}\right)=0$,

with the adiabatic index $\gamma=5 / 3$.

For a gyrotropic collisionless plasma, one has

$\mathbf{P}=P_{\perp} \mathbf{I}+\left(P_{\|}-P_{\perp}\right) \widehat{\boldsymbol{e}}_{\mathrm{B}} \widehat{\boldsymbol{e}}_{\mathrm{B}}$

and

$\nabla \cdot \mathbf{P}=\nabla_{\perp} P_{\perp}+\nabla_{\|} P_{\|}-\left(P_{\perp}-P_{\|}\right)\left(c-\frac{\nabla_{\|} B}{B}\right)$ 
(e.g. Krall and Trivelpiece, 1973; Siscoe, 1983). If the heat flux along magnetic field lines vanishes, the perpendicular and parallel thermal pressures satisfy the double-adiabatic equations of state,

$\frac{D}{D t}\left(\frac{P_{\perp}}{\rho B}\right)=0$

and

$$
\frac{D}{D t}\left(\frac{P_{\|} B^{2}}{\rho^{3}}\right)=0
$$

(Chew et al., 1956). However, in general the heat flux along field lines does not vanish (see introduction), and therefore the pressure equations cannot be written in the form of simple closure equations that are correct in the most general case.

Nevertheless, it is possible to obtain correct expressions for the pressure equations in the particular case of lowfrequency linear waves. The way to proceed is to go back to the gyro-kinetic equation of each plasma species (denoted with a subscript $s$ in the following, whenever necessary to avoid a possible ambiguity),

$$
\frac{\partial f}{\partial t}+\dot{\boldsymbol{r}}_{\mathrm{g}} \cdot \nabla f+\dot{v}_{\perp} \frac{\partial f}{\partial v_{\perp}}+\dot{v}_{\|} \frac{\partial f}{\partial v_{\|}}=0
$$

where $f\left(t, \boldsymbol{r}_{\mathrm{g}}, v_{\perp}, v_{\|}\right)$is the distribution function of the considered plasma species averaged over gyromotion, $\boldsymbol{r}_{\mathrm{g}}$ is the guiding center position vector, $v_{\perp}$ the gyration speed, and $v_{\|}$ the field-aligned speed. The Lagrangian derivatives of the last three variables are given by

$\dot{\boldsymbol{r}}_{\mathrm{g}}=\boldsymbol{V}_{\perp}+v_{\|} \widehat{\boldsymbol{e}}_{\mathrm{B}}$

(consistent with the frozen-in approximation inherent in Eq. 3),

$\dot{v}_{\perp}=\frac{1}{2} \frac{\dot{B}}{B} v_{\perp}$

(as implied by the conservation of the magnetic moment, $\left.\mu \equiv\left(\frac{1}{2} m v_{\perp}^{2}\right) / B\right)$, and

$\dot{v}_{\|}=\frac{q}{m} E_{\|}-\frac{\frac{1}{2} v_{\perp}^{2}}{B} \frac{\partial B}{\partial s}+g_{\|}-\left(2 \boldsymbol{\Omega} \times \boldsymbol{V}_{\perp}\right)_{\|}+\boldsymbol{V}_{\perp} \cdot \dot{\hat{\boldsymbol{e}}}_{\mathrm{B}}$

(parallel component of the equation of motion, where the parallel electric field, $E_{\|}$, is to be inferred from the chargeneutrality condition) (adapted from Northrop, 1963). The overall idea is to perturb, linearize, and Fourier-transform the gyro-kinetic equation so as to obtain the expression of the perturbation in $f$, then take its second-order moments to deduce the expressions of the perturbations in $P_{\perp}$ and $P_{\|}$for each plasma species, and finally sum over all the species. Strictly speaking, however, explicit expressions can be obtained only if the equilibrium parameters have no fieldaligned gradient. We are thus led to make this restrictive assumption (see first paragraph of Sect. 3), knowing that it automatically excludes all phenomena associated with bounce resonance.

\section{Dispersion relation}

Our purpose is to understand the physical behavior of lowfrequency linear waves occurring in a stratified and rotating magnetized plasma. To be able to proceed analytically and to keep the formalism at a reasonably simple level, we start from the assumption that in the equilibrium state (denoted by a subscript 0 ) the effective gravity, $g$, and the magnetic curvature vector, $\boldsymbol{c}_{0}$, are parallel to each other and perpendicular to the magnetic field, $\boldsymbol{B}_{0}$. Accordingly, the equilibrium parameters are constant along field lines and vary only in the direction $\widehat{\boldsymbol{e}}_{\mathrm{g}}$. In addition, we restrict our attention to small-scale perturbations, i.e. perturbations with a wavelength much shorter than the typical scale height of the equilibrium, $H_{\mathrm{eq}}$.

To derive the dispersion relation of our low-frequency, small-scale, linear waves, we follow the usual procedure. Namely, we perturb the governing equations about the equilibrium state, we linearize in the small amplitude of the wave perturbations, we Fourier-transform the linearized equations by assigning perturbations a spatio-temporal dependence in $\exp [i(\boldsymbol{k} \cdot \boldsymbol{r}-\omega t)]$ with $\boldsymbol{k}$ real, and we cancel the determinant of the coefficient matrix.

The final result is a pseudo sixth-degree equation for the wave frequency, $\omega$, which can be written in the form

$$
\begin{aligned}
\omega^{6}- & \left(V_{A}^{2}+\gamma_{\perp_{\perp}} C_{\perp}^{2}\right) k_{\perp}^{2}+\left(2 \mathcal{F}+\gamma_{\|\|} C_{\|}^{2}\right) k_{\|}^{2} \\
& \left.+\omega_{0}^{2}+4 \Omega^{2}\right] \omega^{4} \\
+ & 2 \mathcal{G}\left(2 \Omega_{\perp} \cdot \boldsymbol{k}_{\perp}\right) \frac{k_{\mathrm{t}}}{k_{\perp}^{2}} k_{\|} \omega^{3} \\
+ & \left\{\left(V_{A}^{2}+\gamma_{\perp \perp} C_{\perp}^{2}\right) \mathcal{F}+\gamma_{\|\|} C_{\|}^{2} \mathcal{M}\right] k_{\perp}^{2} k_{\|}^{2} \\
& +\mathcal{F}\left(\mathcal{F}+2 \gamma_{\|\|} C_{\|}^{2}\right) k_{\|}^{4} \\
& +\left(V_{A}^{2}+\gamma_{\perp \perp} C_{\perp}^{2}\right)\left(\omega_{0}^{2} k_{\mathrm{t}}^{2}+\left(2 \boldsymbol{\Omega}_{\perp} \cdot \boldsymbol{k}_{\perp}\right)^{2}\right) \\
& +\left(\mathcal{F} 4 \Omega_{\perp}^{2}+\gamma_{\|\|} C_{\|}^{2} 4 \Omega_{\|}^{2}\right) k_{\|}^{2} \\
& \left.+2 \gamma_{\perp \|} C_{\perp}^{2}\left(2 \boldsymbol{\Omega}_{\perp} \cdot \boldsymbol{k}_{\perp}\right) 2 \Omega_{\|} k_{\|}\right\} \omega^{2} \\
-2 & \left(V_{A}^{2}+\gamma_{\perp \perp} C_{\perp}^{2}\right) \mathcal{G}\left(2 \boldsymbol{\Omega}_{\perp} \cdot \boldsymbol{k}_{\perp}\right) k_{\mathrm{t}} k_{\|} \omega \\
- & \gamma_{\|\|} C_{\|}^{2}\left[\mathcal{F}\left(\mathcal{M} k_{\perp}^{2}+\mathcal{F} k_{\|}^{2}\right) k_{\|}^{2}+\mathcal{M}\left(\omega_{0}^{2}-\omega_{1}^{2}\right) k_{\mathrm{t}}^{2}\right] k_{\|}^{2} \\
= & 0
\end{aligned}
$$

where the new parameters are defined as follows: $\widehat{\boldsymbol{e}}_{\mathrm{t}}$ is the unit vector in the direction perpendicular to both $\boldsymbol{B}_{0}$ and $\boldsymbol{g}, V_{A}^{2}=\left(2 P_{\mathrm{M} 0} / \rho_{0}\right)$ is the Alfvén speed squared, $C_{\perp}^{2}=\left(P_{\perp 0} / \rho_{0}\right)$ and $C_{\|}^{2}=\left(P_{\| 0} / \rho_{0}\right)$ are the global perpendicular and parallel sound speeds squared (as opposed to $C_{s \perp}^{2}=\left(P_{s \perp 0} / \rho_{0}\right)$ and $C_{s \|}^{2}=\left(P_{s \| 0} / \rho_{0}\right)$, which denote the partial perpendicular and parallel sound speeds squared of species $s$ ),

$$
\mathcal{F}=V_{A}^{2}+C_{\perp}^{2}-C_{\|}^{2}
$$


and

$$
\mathcal{M}=V_{A}^{2}+\gamma_{\perp_{\perp}} C_{\perp}^{2}-\frac{\gamma_{\perp_{\|}}^{2} C_{\perp}^{4}}{\gamma_{\|\|} C_{\|}^{2}}
$$

are the characteristic speeds squared governing the firehose and mirror instabilities, respectively, in a uniform plasma,

$$
\begin{aligned}
\omega_{0}^{2} & =\left(\frac{\nabla \rho_{0}}{\rho_{0}}-\boldsymbol{c}_{0}\right) \cdot \boldsymbol{g} \\
& +\left[\frac{\nabla\left(P_{\mathrm{M} 0}-P_{\| 0}\right)}{\rho_{0}}+\gamma_{\|\|} C_{\|}^{2} \boldsymbol{c}_{0}\right] \cdot \boldsymbol{c}_{0} \\
& -\frac{g_{*}^{2}}{V_{A}^{2}+\gamma_{\perp \perp} C_{\perp}^{2}}
\end{aligned}
$$

is the generalized magnetic Rayleigh-Taylor (or magnetic Brunt-Väisälä) frequency squared, which together with

$\omega_{1}^{2}=\frac{\left(V_{A}^{2}+\gamma_{\perp_{\perp}} C_{\perp}^{2}\right) \mathcal{G}^{2}}{\gamma_{\|\|} C_{\|}^{2} \mathcal{M}}$,

govern the stratification (interchange and Parker) instabilities,

$g_{*}=\left[\boldsymbol{g}+\left(V_{A}^{2}-\gamma_{\|_{\perp}} C_{\|}^{2}\right) \boldsymbol{c}_{0}\right] \cdot \widehat{\boldsymbol{e}}_{\mathrm{g}}$

and

$\mathcal{G}=\left(\boldsymbol{g}-\gamma_{\|\|} C_{\|}^{2} \boldsymbol{c}_{0}\right) \cdot \widehat{\boldsymbol{e}}_{\mathrm{g}}-\frac{\gamma_{\perp_{\|}} C_{\perp}^{2}}{V_{A}^{2}+\gamma_{\perp_{\perp}} C_{\perp}^{2}} g_{*}$

are working variables with dimension of gravity, and $\gamma_{\perp_{\perp}}$, $\gamma_{\perp_{\|}}, \gamma_{\|_{\perp}}, \gamma_{\|_{\|}}$are the polytropic indices defined through the equations relating the normalized Lagrangian perturbations in perpendicular and parallel thermal pressures to the perpendicular and parallel spatial convergences:

$$
\begin{aligned}
& \frac{D P_{\perp}}{P_{\perp 0}}=-\gamma_{\perp_{\perp}}(\nabla \cdot \delta \boldsymbol{r})_{\perp}-\gamma_{\perp_{\|}}(\nabla \cdot \delta \boldsymbol{r})_{\|} \\
& \frac{D P_{\|}}{P_{\| 0}}=-\gamma_{\|_{\perp}}(\nabla \cdot \delta \boldsymbol{r})_{\perp}-\gamma_{\|_{\|}}(\nabla \cdot \delta \boldsymbol{r})_{\|},
\end{aligned}
$$

with $\delta \boldsymbol{r}$ the Lagrangian displacement vector, $(\boldsymbol{\nabla} \cdot \delta \boldsymbol{r})_{\|} \equiv$ $\left(\widehat{\boldsymbol{e}}_{\mathrm{B}_{0}} \cdot \nabla \delta \boldsymbol{r}\right) \cdot \widehat{\boldsymbol{e}}_{\mathrm{B}_{0}}$ the parallel convergence, and $(\nabla \cdot \delta \boldsymbol{r})_{\perp} \equiv$ $(\nabla \cdot \delta \boldsymbol{r})-(\boldsymbol{\nabla} \cdot \delta \boldsymbol{r})_{\|}$the perpendicular convergence. Note that our definitions of the polytropic indices differ from those introduced by Belmont and Mazelle (1992) and by Hau and Sonnerup (1993), both of whom used only two indices, $\gamma_{\perp}$ and $\gamma_{\|}$. The former defined their indices via the relations $\left(D P_{\perp} / P_{\perp 0}\right)=-\gamma_{\perp}(\nabla \cdot \delta \boldsymbol{r})$ and $\left(D P_{\|} / P_{\| 0}\right)=$ $-\gamma_{\|}(\nabla \cdot \delta \boldsymbol{r})$, whereas the latter adopted relations similar to ours with $\gamma_{\perp_{\perp}}=\gamma_{\perp}, \gamma_{\|_{\|}}=\gamma_{\|}$, and $\gamma_{\perp_{\|}}=\gamma_{\|_{\perp}}=1$.

The values or expressions of our polytropic indices depend on the formalism adopted to derive the dispersion relation. In isotropic-adiabatic MHD (Sect. 3.1) and in gyrotropicdouble-adiabatic MHD (Sect. 3.2), the polytropic indices take on constant values, while in our exact mixed MHDkinetic theory (Sect. 3.3), they are given by complicated functions of the wave frequency, $\omega$, its parallel wavenumber, $k_{\|}$, and the equilibrium parameters.

In all three formalisms, the cross polytropic indices, $\gamma_{\perp_{\|}}$ and $\gamma_{\| \perp}$, are related through Eq. (A8). In view of its general validity, this relation was utilized to simplify the expressions of the parameters $\mathcal{M}, \omega_{0}^{2}$, and $\mathcal{G}$, as well as the form of the dispersion relation.

\subsection{Isotropic - adiabatic MHD}

In isotropic-adiabatic MHD, the perpendicular and parallel thermal pressures are equal, not only in the equilibrium state $\left(P_{\perp 0}=P_{\| 0}=P_{0}\right.$ or, equivalently, $C_{\perp}^{2}=C_{\|}^{2}=C_{0}^{2}$ ), but also in the perturbed state $\left(D P_{\perp}=D P_{\|}\right)$, and the four polytropic indices have the same value :

$\gamma_{\perp_{\perp}}=\gamma_{\perp_{\|}}=\gamma_{\|_{\perp}}=\gamma_{\|_{\|}}=\gamma=5 / 3$.

Defining the isotropic sound speed in the usual manner, i.e. by $C_{s}^{2}=\left(\gamma P_{0} / \rho_{0}\right)$, one immediately finds $\gamma_{\perp_{\perp}} C_{\perp}^{2}=$ $\gamma_{\perp \|} C_{\perp}^{2}=\gamma_{\|\|_{\perp}} C_{\|}^{2}=\gamma_{\|} C_{\|}^{2}=C_{s}^{2}$, and hence $\mathcal{F}=\mathcal{M}=V_{A}^{2}$. With the above identities, it is easily verified that our full dispersion relation (Eq. 16) is equivalent to that derived by Ferrière et al. (1999) (see their Eq. 27).

\subsection{Gyrotropic - double-adiabatic MHD}

In double-adiabatic MHD, the perpendicular and parallel thermal pressures are generally different, and they evolve according to two different equations (Eqs. 10 and 11), from which the values of the polytropic indices can be deduced. Remarking that the normalized density and field-strength perturbations are related to the perpendicular and parallel spatial convergences through

$\frac{D \rho}{\rho_{0}}=-(\nabla \cdot \delta \boldsymbol{r})_{\perp}-(\nabla \cdot \delta \boldsymbol{r})_{\|}$

and

$\frac{D B}{B_{0}}=-(\nabla \cdot \delta \boldsymbol{r})_{\perp}$,

it is straightforward to obtain

$\gamma_{\perp_{\perp}}=2, \quad \gamma_{\|_{\|}}=3, \quad \gamma_{\perp_{\|}}=\gamma_{\|_{\perp}}=1$.

These values are those expected from basic thermodynamics, in the sense that a pure perpendicular convergence acts in two dimensions on the perpendicular temperature $\left(\gamma_{\perp_{\perp}}=\right.$ 2) and has no effect on the parallel temperature $\left(\gamma_{\|_{\perp}}=1\right)$, while a pure parallel convergence acts in one dimension on the parallel temperature $\left(\gamma_{\|_{\|}}=3\right)$ and has no effect on the perpendicular temperature $\left(\gamma_{\perp_{\|}}=1\right)$.

\subsection{Gyrotropic - exact MHD-kinetic theory}

In our exact mixed MHD-kinetic formalism, the polytropic indices no longer take on constant values. Instead, they depend on the equilibrium parameters (both the macroscopic parameters and the distribution functions of all the plasma 
components) as well as on the parallel phase speed, $\left(\omega / k_{\|}\right)$, of the considered wave. The latter dependence arises from the Landau factor $\left(\omega-k_{\|} v_{\|}\right)$appearing in the expressions of the perturbed distribution functions. Physically, this factor represents the Doppler-shifted frequency felt by particles with parallel velocity $v_{\|}$and lies at the root of parallel Landau damping.

The full expressions of the polytropic indices in the case of bi-Maxwellian distributions were derived by André and Ferrière (2004) and can be found (in a more compact form) in the Appendix. These unwieldy expressions are evidently non-adiabatic, for the physical reasons explained in detail by Ferrière and André (2002). In brief, all non-adiabatic behaviors can be traced back to the parallel motion of particles. For $P_{\perp}$, the culprit is the magnetic mirror force, which couples the perpendicular microscopic (gyration) motion of particles to their parallel motion. For $P_{\|}$, the $v_{\|}$-dependence of the Doppler-shifted frequency causes particles with different $v_{\|}$ to respond differently to the parallel force of the wave.

For the following, let us just point out that in the limit $\left(\omega / k_{\|}\right) \rightarrow \infty$, our polytropic indices take on the doubleadiabatic values given by Eq. (28). Physically, in this limit, (1) the magnetic mirror force becomes negligible, so that $P_{\perp}$ regains an adiabatic behavior; and (2) the $v_{\|}$-dependence of the parallel velocity perturbation is very weak and can be linearized, so that the resulting divergence in velocity space, which by virtue of Liouville's theorem is equivalent to a convergence in physical space, and the net increase in the mean parallel energy of particles (Fermi acceleration) ${ }^{1}$ have the values expected for an adiabatic one-dimensional parallel compression.

In the remainder of this paper, we will assume that the equilibrium distribution functions are bi-Maxwellian.

\section{Waves in a uniform plasma}

If the rotation rate, $\Omega$, and the stratification parameters, $\omega_{0}^{2}$, $\omega_{1}^{2}$, and $\mathcal{G}$, are set to zero, the complete dispersion relation of low-frequency linear waves (Eq. 16) reduces to

$$
\begin{aligned}
& \left\{\omega^{2}-\mathcal{F} k_{\|}^{2}\right\} \\
\times\left\{\omega^{4}-\right. & {\left[\left(V_{A}^{2}+\gamma_{\perp_{\perp}} C_{\perp}^{2}\right) k_{\perp}^{2}+\left(\mathcal{F}+\gamma_{\|\|} C_{\|}^{2}\right) k_{\|}^{2}\right] \omega^{2} } \\
+ & \left.\gamma_{\|\|} C_{\|}^{2}\left(\mathcal{M} k_{\perp}^{2}+\mathcal{F} k_{\|}^{2}\right) k_{\|}^{2}\right\}=0 .
\end{aligned}
$$

The first factor in Eq. (29) represents the shear Alfvén mode. This mode has $\delta \boldsymbol{r} \perp\left(\boldsymbol{B}_{0}, \boldsymbol{k}\right)$, so that it is noncompressive and thus unaffected by the exact form of the equations of state. This is why its dispersion relation,

$\omega^{2}=\mathcal{F} k_{\|}^{2}$,

${ }^{1}$ Here it is assumed that the equilibrium distribution functions are symmetric in $v_{\|}$. Then the net energy increase is only due to the small difference between the energy gained by particles with $v_{\|}>0$ and the energy lost by particles with $v_{\|}<0$. is independent of the polytropic indices. It directly follows from Eq. (30) that the Alfvén mode is stable (and then purely oscillatory) if and only if

$\mathcal{F} \geq 0$.

When $\mathcal{F}<0$, the Alfvén mode is unstable, at all $k_{\|}$, to the so-called firehose instability.

The second factor in Eq. (29) represents the compressional modes, for which $\delta \boldsymbol{r}$ lies in the plane $\left(\boldsymbol{B}_{0}, \boldsymbol{k}\right)$. Their dispersion relation,

$$
\begin{aligned}
\omega^{4} & -\left[\left(V_{A}^{2}+\gamma_{\perp_{\perp}} C_{\perp}^{2}\right) k_{\perp}^{2}+\left(\mathcal{F}+\gamma_{\|\|} C_{\|}^{2}\right) k_{\|}^{2}\right] \omega^{2} \\
+ & \gamma_{\|\|} C_{\|}^{2}\left(\mathcal{M} k_{\perp}^{2}+\mathcal{F} k_{\|}^{2}\right) k_{\|}^{2}=0,
\end{aligned}
$$

constitutes a pseudo quadratic equation for $\omega^{2}$. When the polytropic indices have constant values (as in isotropicadiabatic and gyrotropic-double-adiabatic MHD), Eq. (32) is a true quadratic equation for $\omega^{2}$, and its two solutions, ordered according to their phase speed, correspond to the fast and slow magnetosonic modes. When the polytropic indices are functions of $\left(\omega / k_{\|}\right)$(as in our exact mixed MHDkinetic formalism), Eq. (32) possesses a third physical solution, known in kinetic theory as the mirror mode.

The limiting behaviors of compressional modes at perpendicular and parallel wave vectors are easily inferred from Eq. (32). For $\boldsymbol{k} \perp \boldsymbol{B}_{0}\left(k_{\|}=0\right)$, the fast mode satisfies

$\omega^{2}=\left[V_{A}^{2}+\gamma_{\perp_{\perp}}(\infty) C_{\perp}^{2}\right] k_{\perp}^{2}$,

with $\gamma_{\perp_{\perp}}(\infty)=5 / 3$ in isotropic-adiabatic MHD (see Eq. 25) and $\gamma_{\perp_{\perp}}(\infty)=2$ both in double-adiabatic MHD (see Eq. 28) and for bi-Maxwellian distributions in the mixed formalism (see Sect. 3.3); as for the slow and (if relevant) mirror modes, they satisfy

$\omega^{2}=0$.

For $\boldsymbol{k} \| \boldsymbol{B}_{0}\left(k_{\perp}=0\right)$, one of the compressional modes becomes purely Alfvénic with

$\omega^{2}=\mathcal{F} k_{\|}^{2}$,

another mode becomes purely acoustic with

$\omega^{2}=\left(\gamma_{\|\|} C_{\|}^{2}\right) k_{\|}^{2}$

where $\gamma_{\|\|}=5 / 3$ in isotropic-adiabatic MHD (see Eq. 25), $\gamma_{\|\|}=3$ in double-adiabatic MHD (see Eq. 28), and $\gamma_{\|\|}=\gamma_{\|\|}\left(\omega / k_{\|}\right)$has a value generally comprised between 1 (isothermal value) and 3 (adiabatic value) in the mixed formalism; finally (if relevant), the last mode is infinitely damped with

$\omega=-i|\infty|$

Let us now inquire into the stability of compressional modes. In isotropic-adiabatic and gyrotropic-doubleadiabatic MHD, where the polytropic indices have constant 
and positive values, the two solutions of Eq. (32) are stable (with $\omega^{2}$ real and positive) if and only if

$\mathcal{M}(0) k_{\perp}^{2}+\mathcal{F} k_{\|}^{2} \geq 0$

for all $\boldsymbol{k}$, i.e. if and only if

$\mathcal{F} \geq 0$ and $\mathcal{M}(0) \geq 0$.

The reason why we assigned an argument of value 0 to $\mathcal{M}$ is because with $\mathcal{M}$ taken at $\left(\omega / k_{\|}\right)=0$, Eqs (38) and (39) remain valid in the exact mixed formalism (at least for biMaxwellian distributions; see Ferrière and André, 2002).

By combining Eqs. (31) and (39), we may conclude that the necessary and sufficient condition for all low-frequency modes (Alfvén and compressional) to be stable at all $\boldsymbol{k}$ is given by Eq. (39).

\subsection{Isotropic - adiabatic MHD}

When $C_{\perp}^{2}=C_{\|}^{2}$ and the polytropic indices obey Eq. (25), we saw in Sect. 3.1 that $\mathcal{F}=\mathcal{M}=V_{A}^{2}$. Under these conditions, Eqs. (30) and (32) reduce to the standard dispersion relations of the shear Alfvén mode and of the fast and slow magnetosonic modes, respectively. All three modes are stable and purely oscillatory at all $\boldsymbol{k}$, in agreement with Eq. (39) being automatically satisfied here. Moreover, the Alfvén mode always has a phase speed intermediate between those of the fast and slow magnetosonic modes.

\subsection{Gyrotropic - double-adiabatic MHD}

When the polytropic indices take on the values given by Eq. (28), one recovers the dispersion relations derived by Kato et al. (1966) and by Abraham-Shrauner (1967). Here, $\mathcal{F}$ (see Eq. 17) and

$\mathcal{M}=V_{A}^{2}+2 C_{\perp}^{2}-\frac{C_{\perp}^{4}}{3 C_{\|}^{2}}$

(see Eq. 18) can become negative, with the consequence that some of the double-adiabatic modes can turn unstable. Nonetheless, $\omega^{2}$ always remains real, so that all modes are either purely oscillatory or purely growing/decaying. The phase speed ordering found in isotropic-adiabatic MHD still holds for the fast mode, but not necessarily between the Alfvén and slow modes.

The stability condition of the Alfvén mode is, as in the other formalisms, given by Eq. (31). The fast mode, which has the highest phase speed, is always stable. The slow mode is stable if and only if Eq. (39) with $\mathcal{M}(0)=\mathcal{M}$ is satisfied. When $\mathcal{F}<0$, the slow mode is firehose unstable at large enough $k_{\|}\left[k_{\|}^{2}>(\mathcal{M} /|\mathcal{F}|) k_{\perp}^{2}\right]$, and when $\mathcal{M}<0$, the slow mode is said to be mirror unstable at large enough $k_{\perp}\left[k_{\perp}^{2}>\right.$ $\left.(\mathcal{F} /|\mathcal{M}|) k_{\|}^{2}\right]$. The denomination of mirror instability is a little misleading in the present context, since it does not refer to the mirror mode (as it does in the exact mixed formalism; see Sect. 4.3).
The above conclusions also apply to the double-polytropic MHD model of Hau and Sonnerup (1993), provided the expression of $\mathcal{M}$ be given by the double-polytropic generalization of Eq. (40), namely, Eq. (18) with $\gamma_{\perp_{\perp}}=\gamma_{\perp}, \gamma_{\|\|}=\gamma_{\|}$, and $\gamma_{\perp_{\|}}=\gamma_{\|_{\perp}}=1$ (double-polytropic generalization of Eq. 28). In particular, Wang and Hau (2003), who focused on the firehose instability, showed that this instability in doublepolytropic MHD can affect both the noncompressive Alfvén mode (at all $\boldsymbol{k}$ ) and the compressional slow mode (above some critical value of $k_{\|} / k_{\perp}$ ), and they derived stability criteria identical to those we would obtain here with the doublepolytropic indices.

\subsection{Gyrotropic - exact MHD-kinetic theory}

In the mixed formalism, the Alfvén mode has the exact same properties as in double-adiabatic MHD. As explained before, this is because its noncompressive nature makes it insensitive to the values of the polytropic indices. In contrast, compressional modes generally have complex frequencies, such that oscillatory solutions can now be damped (though still not growing). The normal phase speed ordering between fast and slow modes remains valid for $k_{\|} \rightarrow 0$ (compare Eqs. 33 and 34), but may undergo an inversion away from this limit. Accordingly, it is no longer true that the mode becoming unstable when $\mathcal{F}<0$ or $\mathcal{M}(0)<0$ is automatically the slow mode.

The stability properties of compressional modes in the case of bi-Maxwellian distributions were investigated by Ferrière and André (2002). Expressed in terms of the firehose $(\mathcal{F})$ and mirror $(\mathcal{M})$ parameters introduced in Sect. 3, they can be summarized as follows. The stability of the fast mode is governed by the sign of $\mathcal{F}$ (unless both $\mathcal{F}$ and $\mathcal{M}(0)$ happen to be negative, in which case it is the sign of $\mathcal{M}(0)$ that dictates the nature of the unstable mode): if $\mathcal{F} \geq 0$, the fast mode is oscillatory and weakly damped at all $\boldsymbol{k}$; otherwise, it is firehose unstable at sufficiently large $k_{\|}$ $\left[k_{\|}^{2}>(\mathcal{M}(0) /|\mathcal{F}|) k_{\perp}^{2}\right]$. The slow mode is always oscillatory and strongly damped (except right at $k_{\|}=0$, where it has $\omega=0$ ). The mirror mode is always nonoscillatory, and its stability is governed by the sign of $\mathcal{M}(0)$ : if $\mathcal{M}(0) \geq 0$, the mirror mode is purely decaying at all $\boldsymbol{k}$; otherwise, it is mirror unstable at sufficiently large $k_{\perp}\left[k_{\perp}^{2}>(\mathcal{F} /|\mathcal{M}(0)|) k_{\|}^{2}\right]$.

A summary of the nature and wave-vector range of the unstable modes (compressional or not) as functions of the parameters $\mathcal{F}$ and $\mathcal{M}(0)$ is provided in Table 1.

Let us close this section with a quantitative comparison between the expression of $\mathcal{M}$ obtained in double-adiabatic MHD (Eq. 40) and the expression of $\mathcal{M}(0)$ obtained for a two-component ion-electron bi-Maxwellian plasma in the exact mixed formalism. By introducing into Eq. (18) the expressions of the polytropic indices given in the Appendix and taken at $\left(\omega / k_{\|}\right)=0$, we find after some algebra

$$
\mathcal{M}(0)=V_{A}^{2}+2 C_{\perp}^{2}-\left(\frac{C_{\perp}^{4}}{C_{\|}^{2}}+\sum_{s} \frac{C_{s \perp}^{4}}{C_{s \|}^{2}}\right) .
$$


Table 1. Nature and wave-vector range of the unstable modes in a uniform plasma, as functions of the firehose $(\mathcal{F})$ and mirror $(\mathcal{M})$ parameters defined by Eqs. (17) and (18), when the equilibrium distribution functions are bi-Maxwellian. The argument of $\mathcal{M}$ refers to the parallel phase speed, $\left(\omega / k_{\|}\right)$.

\begin{tabular}{lccc}
\hline Domains of parameter space & Unstable modes & Instability ranges \\
\hline $\mathcal{F} \geq 0$ & $\mathcal{M}(0) \geq 0$ & \\
$\mathcal{F} \geq 0$ & $\mathcal{M}(0)<0$ & mirror & $\mathcal{F} k_{\|}^{2}<|\mathcal{M}(0)| k_{\perp}^{2}$ \\
$\mathcal{F}<0$ & $\mathcal{M}(0) \geq 0$ & Alfvén & all $k_{\|}$ \\
& & fast & $|\mathcal{F}| k_{\|}^{2}>\mathcal{M}(0) k_{\perp}^{2}$ \\
$\mathcal{F}<0$ & $\mathcal{M}(0)<0$ & Alfvén & all $k_{\|}$ \\
& & mirror & all $k_{\|}$ \\
\hline
\end{tabular}

If only one component contributes to the thermal pressures or if both components have the same ratio $\left(C_{s \perp}^{2} / C_{s \|}^{2}\right)$, the term within parentheses in Eq. (41) reduces to $\left(2 C_{\perp}^{4} / C_{\|}^{2}\right)$, which exceeds the corresponding term in Eq. (40) by a factor of 6 . This factor of 6 discrepancy was already pointed out by Abraham-Shrauner (1967), who made the implicit assumption $P_{e \perp}, P_{e \|} \rightarrow 0$, and by Tajiri (1967), who assumed $\left(C_{i \perp}^{2} / C_{i \|}^{2}\right)=\left(C_{e \perp}^{2} / C_{e \|}^{2}\right)$.

Altogether then, double-adiabatic MHD correctly predicts the properties of the Alfvén mode as well as the firehose instability criterion. However, it fails for compressional modes, particularly with regard to the mirror instability threshold, which is found much more difficult to reach than in the exact mixed formalism.

\section{Waves in a stratified plasma}

In a stratified and rotating plasma, the Alfvén mode no longer decouples from the compressional modes, and the frequency squared of each mode is modified by an amount $\Delta \omega^{2} \sim$ $\left(\omega_{0}^{2}, \Omega^{2}\right) \sim\left(V_{A}^{2}, C_{\perp}^{2}, C_{\|}^{2}\right) / H_{\text {eq }}^{2}$ (see Eq. 19). At short wavelengths $\left(1 / k \ll H_{\text {eq }}\right)$, this corresponds to a small relative modification $\left(\Delta \omega^{2} \ll \omega^{2}\right)$, except for the Alfvén, slow, and (if relevant) mirror modes at $k_{\|} \ll k$, which would have $\omega^{2} \ll\left(V_{A}^{2}, C_{\perp}^{2}, C_{\|}^{2}\right) k^{2}$ in a uniform plasma (see Eqs. 30 and 34). This means that the only small-scale modes significantly affected by stratification and rotation are the Alfvén, slow, and (if relevant) mirror modes at nearly perpendicular wave vectors. Adopting and generalizing the terminology of Newcomb (1961), who studied the special case of a nonrotating plasma with a straight magnetic field in the framework of isotropic-adiabatic MHD, we will give these modes the name of quasi-interchange modes.

The physical characteristics of quasi-interchange modes were discussed both in isotropic-adiabatic MHD (Ferrière et al., 1999) and in the gyrotropic-exact MHD-kinetic for- malism (Ferrière and André, 2003). For our present purposes, it suffices to note that quasi-interchange modes are quasi-incompressible both across and along magnetic field lines and that they leave the total perpendicular pressure, $P_{\mathrm{M}}+P_{\perp}$, unperturbed up to first order in the expansion parameter $\varepsilon \equiv 1 /\left(k_{\perp} H_{\mathrm{eq}}\right)$.

As in Newcomb's (1961) original paper, quasi-interchange modes come into two types, which can be distinguished by their behavior in the limit $k_{\|} \rightarrow 0$. In this limit, the two physical solutions of Eq. (16) that would have $\omega^{2} \rightarrow 0$ in a uniform plasma are

$\omega^{2}=\frac{\omega_{0}^{2}(\infty) k_{\mathrm{t}}^{2}+\left(2 \boldsymbol{\Omega}_{\perp} \cdot \boldsymbol{k}_{\perp}\right)^{2}}{k_{\perp}^{2}}$,

corresponding to the type 1 mode, and

$\omega^{2}=0$,

corresponding to the type 2 mode. The third solution is approximately Eq. (33), which corresponds to the fast mode.

The type 1 mode is a direct generalization to a magnetized medium of the classic gravity or Rayleigh-Taylor mode. In the magnetospheric community, it is usually referred to as the interchange mode. It causes magnetic flux tubes, together with their enclosed plasma, to move perpendicular to themselves, under the effect of (gravitational + magnetic) buoyancy. In the absence of rotation $(\boldsymbol{\Omega}=0)$, the type 1 mode has $\delta \boldsymbol{r} \perp \boldsymbol{B}_{0}$ (pure interchange) in the limit $k_{\|} \rightarrow 0$. The type 2 mode represents the second gravity-driven mode in a magnetized medium. In the context of the interstellar medium, is is known as the Parker instability mode. It causes flux tubes to ripple and the plasma to move along them, under the combined action of a thermal pressure gradient and a gravitational force component along the deformed field lines. Independent of the value of the rotation rate, the type 2 mode has $\delta \boldsymbol{r} \| \boldsymbol{B}_{0}$ (pure translation) in the limit $k_{\|} \rightarrow 0$.

Let us now proceed with a stability analysis of the different modes. To keep the problem tractable analytically, we 
will, from now on, restrict our attention to nonrotating plasmas $(\boldsymbol{\Omega}=0)$, for which the full dispersion relation (Eq. 16) becomes a pseudo cubic equation for $\omega^{2}$,

$$
\begin{aligned}
\omega^{6}-[ & \left.\left(V_{A}^{2}+\gamma_{\perp_{\perp}} C_{\perp}^{2}\right) k_{\perp}^{2}+\left(2 \mathcal{F}+\gamma_{\|\|} C_{\|}^{2}\right) k_{\|}^{2}+\omega_{0}^{2}\right] \omega^{4} \\
+ & \left\{\left[\left(V_{A}^{2}+\gamma_{\perp_{\perp}} C_{\perp}^{2}\right) \mathcal{F}+\gamma_{\|\|} C_{\|}^{2} \mathcal{M}\right] k_{\perp}^{2} k_{\|}^{2}\right. \\
& +\mathcal{F}\left(\mathcal{F}+2 \gamma_{\|\|} C_{\|}^{2}\right) k_{\|}^{4} \\
& \left.+\left(V_{A}^{2}+\gamma_{\perp_{\perp}} C_{\perp}^{2}\right) \omega_{0}^{2} k_{\mathrm{t}}^{2}\right\} \omega^{2} \\
- & \gamma_{\|\|} C_{\|}^{2}\left[\mathcal{F}\left(\mathcal{M} k_{\perp}^{2}+\mathcal{F} k_{\|}^{2}\right) k_{\|}^{2}+\mathcal{M}\left(\omega_{0}^{2}-\omega_{1}^{2}\right) k_{\mathrm{t}}^{2}\right] k_{\|}^{2} \\
= & 0 .
\end{aligned}
$$

We will tackle the problem from a broader perspective than in our previous papers, considering all the modes contained in the full dispersion relation, instead of focusing from the outset on quasi-interchange modes.

In isotropic-adiabatic and gyrotropic-double-adiabatic MHD, where the polytropic indices have constant and positive values, the three solutions of Eq. (44) are stable (with $\omega^{2}$ real and positive) if and only if the coefficients of $\omega^{4}, \omega^{2}$, and $\omega^{0}$ are, respectively, negative, positive, and negative at all $\boldsymbol{k}$.

\subsection{Isotropic - adiabatic MHD}

With $\mathcal{F}=\mathcal{M}=V_{A}^{2}$ (see Sect. 3.1), the coefficient of $\omega^{4}$ in Eq. (44) is always negative, insofar as $\omega_{0}^{2} \sim$ $\left(V_{A}^{2}, C_{s}^{2}\right) / H_{\mathrm{eq}}^{2} \ll\left(V_{A}^{2}, C_{s}^{2}\right) k^{2}$. The requirements on the coefficients of $\omega^{2}$ and $\omega^{0}$ are equivalent to

$\omega_{0}^{2} \geq 0$

and

$\omega_{0}^{2} \geq \omega_{1}^{2}$

respectively. Since $\omega_{1}^{2}$ is necessarily positive here (see Eq. 20), Eq. (46) is always more stringent than Eq. (45). From this we may conclude that Eq. (46) defines the necessary and sufficient condition for stability against all lowfrequency, small-scale perturbations.

It is noteworthy that Eq. (45) coincides with the stability criterion of the type 1 mode. In view of Eq. (42) (with $\boldsymbol{\Omega}=0$ and $\omega_{0}^{2}(\infty)=\omega_{0}^{2}$ ), this means that the stability of the type 1 mode is dictated by its behavior at $k_{\|} \rightarrow 0$. For comparison, the stability criterion of the type 2 mode reads

$\omega_{0}^{2} \leq 0 \quad$ or $\quad \omega_{0}^{2} \geq \omega_{1}^{2}$

(Ferrière et al., 1999).

Thus, as long as $\omega_{0}^{2}$ exceeds $\omega_{1}^{2}$, both quasi-interchange modes are stable. When $\omega_{0}^{2}$ drops below $\omega_{1}^{2}$, one of the quasiinterchange modes becomes unstable; the unstable mode is initially of type 2 , but it switches to type 1 when $\omega_{0}^{2}$ turns negative.

\subsection{Gyrotropic - double-adiabatic MHD}

With $\mathcal{F}$ and $\mathcal{M}$ a priori allowed to be positive or negative (see Sect. 4.2), the requirement that the coefficients of $\omega^{4}, \omega^{2}$, and $\omega^{0}$ in Eq. (44) be, respectively, negative, positive, and negative at all $\boldsymbol{k}$ turns out to be equivalent to the requirement that both the stability condition in a uniform, gyrotropicdouble-adiabatic plasma (Eq. 39) and the stability condition in a stratified, isotropic-adiabatic plasma (Eq. 46) be satisfied simultaneously. As a reminder, the two parts of Eq. (39) refer to the two possible instabilities due to thermal pressure anisotropies, namely, the firehose and mirror instabilities, whereas Eq. (46) refers to the instabilities due to stratification, i.e. the quasi-interchange instabilities of type 1 (interchange) or type 2 (Parker).

Here, too, Eq. (45) applies to the type 1 mode, but by itself it no longer constitutes a necessary and sufficient condition for stability. Only when $\mathcal{F} \geq 0$ does this remain true; when $\mathcal{F}<0$, Eq. (45) provides only a necessary condition for stability. Likewise, Eq. (47) still applies to the type 2 mode, but only when $\mathcal{F} \geq 0$ and $\mathcal{M} \geq 0$ does it constitute a necessary and sufficient condition for stability; when $\mathcal{F}<0$ or $\mathcal{M}<0$, it provides only a necessary condition. As in isotropic-adiabatic MHD, when $\omega_{0}^{2}$ turns from positive to negative, an unstable mode switches from type 2 to type 1 , and vice-versa.

\subsection{Gyrotropic - exact MHD-kinetic theory}

When the polytropic indices depend on $\left(\omega / k_{\|}\right)$, the derivation of the stability criteria is much more involved. However, it can be shown that the global criteria have the same form as those obtained in double-adiabatic MHD, with the polytropic indices and, hence, $\mathcal{M}, \omega_{0}^{2}$, and $\omega_{1}^{2}$ taken at $\left(\omega / k_{\|}\right)=0$ (see Ferrière and André, 2003; André and Ferrière, 2004). In other words, the necessary and sufficient condition for all low-frequency, small-scale modes to be stable at all $\boldsymbol{k}$ is given by Eq. (39) (criterion against the anisotropy-driven firehose and mirror instabilities) together with

$\omega_{0}^{2}(0) \geq \omega_{1}^{2}(0)$

(additional criterion against the stratification-driven type 1 or type 2 quasi-interchange instabilities).

An important point to realize is that the exact mixed formalism leads to one mode of type 1 and two modes of type 2 , in contrast to the situation prevailing in isotropic-adiabatic and gyrotropic-double-adiabatic MHD, where there is exactly one quasi-interchange mode of each type. The stability condition of the type 1 mode is still dictated by its behavior at $k_{\|} \rightarrow 0$ (Eq. 42 with $\boldsymbol{\Omega}=0$ ) and is, therefore, given by

$\omega_{0}^{2}(\infty) \geq 0$,

which is formally identical to Eq. (45) taken at $\left(\omega / k_{\|}\right) \rightarrow$ $\infty$; this stability condition is always necessary, but only when $\mathcal{F} \geq 0$ is it also sufficient. Similarly, Eq. (47) with the first 
Table 2. Nature and wave-vector range of the unstable modes in a nonrotating stratified plasma, in the different domains of parameter space emerging from our stability analysis, when the equilibrium distribution functions are bi-Maxwellian. The firehose $(\mathcal{F})$ and mirror $(\mathcal{M})$ parameters are defined by Eqs. (17) and (18). The magnetic Rayleigh-Taylor frequency squared, $\omega_{0}^{2}$, and the threshold frequency squared, $\omega_{1}^{2}$, are defined by Eqs. (19) and (20). The argument of $\mathcal{M}, \omega_{0}^{2}$, and $\omega_{1}^{2}$ refers to the parallel phase speed, $\left(\omega / k_{\|}\right)$. Note that $\omega_{0}^{2}(0)$ and $\omega_{1}^{2}(0)$ can both have either sign.

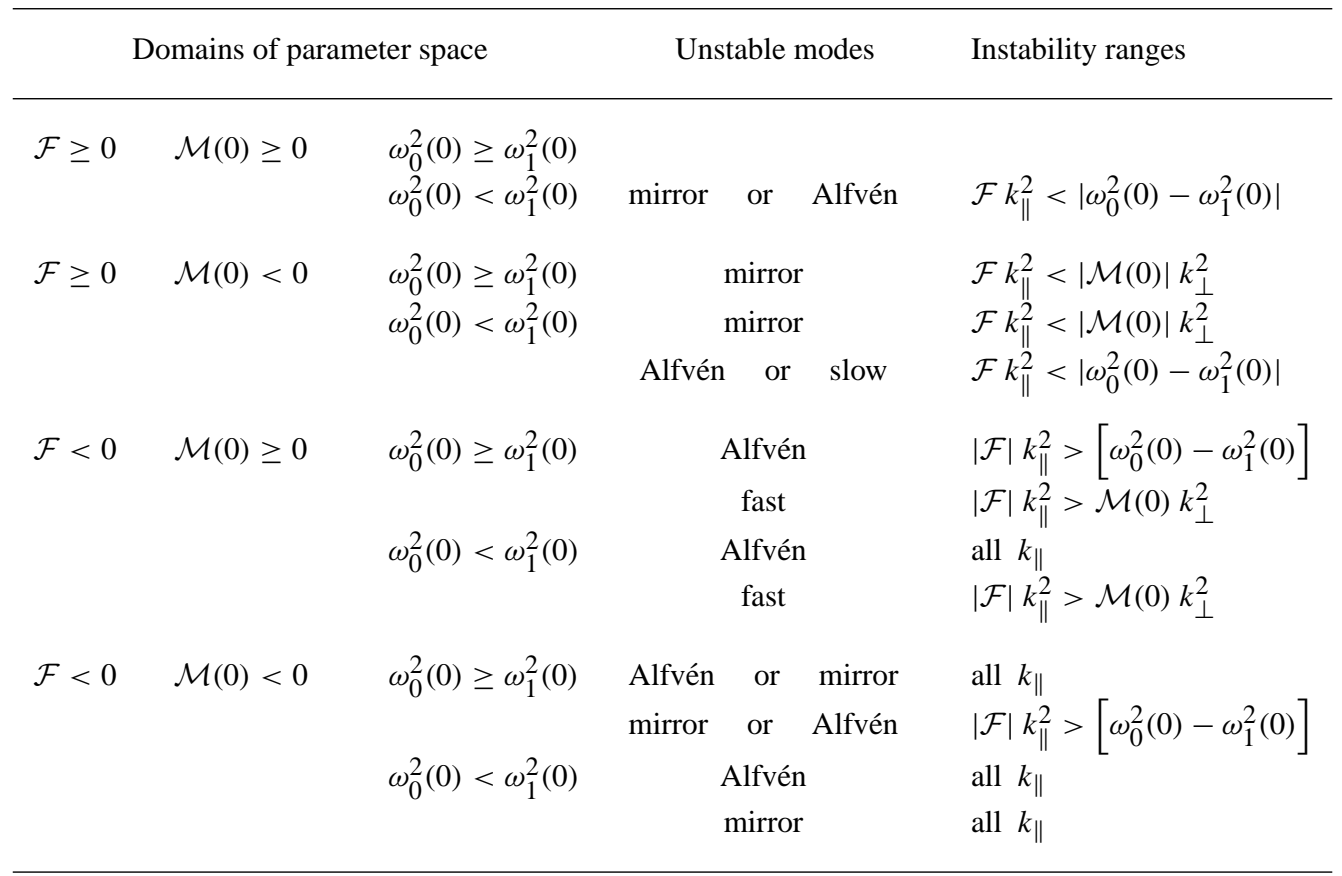

inequality taken at $\left(\omega / k_{\|}\right) \rightarrow \infty$ and the second inequality at $\left(\omega / k_{\|}\right)=0$, i.e.

$\omega_{0}^{2}(\infty) \leq 0 \quad$ or $\quad \omega_{0}^{2}(0) \geq \omega_{1}^{2}(0)$,

yields a stability condition for the type 2 mode, which is always necessary, but which is sufficient only when $\mathcal{F} \geq 0$ and $\mathcal{M} \geq 0$. Once again, a switch in the type of an unstable mode (say, from type 2 to type 1 ) occurs when $\omega_{0}^{2}(\infty)$ changes sign (from positive to negative).

Our stability criteria (Eqs. 39 and 48) define eight separate regions in parameter space, characterized by different stability properties. The nature and wave-vector range of the unstable modes in each of these regions are displayed in Table 2. A comparison with Table 1 clearly shows that stratification affects the stability of certain modes. Changes in stability are possible only when the parameters governing the firehose $(\mathcal{F})$ and quasi-interchange $\left(\omega_{0}^{2}(0)-\omega_{1}^{2}(0)\right)$ instabilities have opposite signs, and they occur solely at parallel wavenumbers smaller than the critical wavenumber $k_{\|}^{*}$ defined by the relation

$\mathcal{F}\left(k_{\|}^{*}\right)^{2}+\left[\omega_{0}^{2}(0)-\omega_{1}^{2}(0)\right]=0$

(belonging, as expected, to the quasi-interchange regime $\left.k_{\|} \ll k\right)$. When $\mathcal{F}>0$ and $\left[\omega_{0}^{2}(0)-\omega_{1}^{2}(0)\right]<0$, stratification destabilizes, below $k_{\|}^{*}$, one mode that would be stable in a uniform medium. Conversely, when $\mathcal{F}<0$ and $\left[\omega_{0}^{2}(0)-\omega_{1}^{2}(0)\right]>0$, stratification stabilizes, below $k_{\|}^{*}$, one mode that would be firehose unstable in a uniform medium.

\section{Conclusions}

After reviewing the existing closure models for collisionless plasmas in the MHD regime, we presented the exact mixed MHD-kinetic model that we ourselves developed to study low-frequency $\left(\omega \ll \Omega_{s}\right)$, small-scale $\left(\lambda \ll H_{\text {eq }}\right)$, linear waves and instabilities in collisionless plasmas, when the ambient medium is either uniform or stratified perpendicular to the background magnetic field. We indicated the steps to take to derive the correct and complete dispersion relation, and we wrote it in a new form, which is more general, better suited both for comparisons with other theories and for stability considerations, and more physically transparent than in our previous papers.

To proceed, we introduced four polytropic indices, $\gamma_{\perp_{\perp}}$, $\gamma_{\perp_{\|}}, \gamma_{\|_{\perp}}$, and $\gamma_{\|_{\|}}$, which describe the frequency-dependent response of the perpendicular and parallel thermal pressures to perpendicular and parallel spatial convergences, and which embody all the relevant information contained in the perturbed closure equations (equations of state). Written in terms of these polytropic indices, our dispersion relation (Eq. 16) is directly applicable to other formalisms, as it suffices to insert in it the values or expressions of 
the polytropic indices appropriate to the desired formalism - e.g. Eq. (25) for isotropic-adiabatic MHD, Eq. (28) for gyrotropic-double-adiabatic MHD, and Eqs. (A6)-(A9) for our gyrotropic-exact MHD-kinetic theory with biMaxwellian distributions. This novelty made it possible for the first time to draw direct and systematic comparisons on the mode properties and the stability criteria obtained in the different formalisms.

We also introduced four stability-governing parameters, $\mathcal{F}, \mathcal{M}, \omega_{0}^{2}$, and $\omega_{1}^{2}$, which greatly facilitated our stability analysis and helped to bring out the physical mechanisms at play. Except for $\mathcal{F}$, these parameters depend on the polytropic indices and, hence, on the adopted formalism. $\mathcal{F}$ and $\mathcal{M}$ (defined by Eqs. 17 and 18) control the firehose and mirror instabilities, respectively, which are the two instabilities arising from thermal pressure anisotropies. The associated necessary and sufficient conditions for stability are given by the two inequalities composing Eq. (39). $\omega_{0}^{2}$ and $\omega_{1}^{2}$ (defined by Eqs. 19 and 20) control the quasi-interchange instabilities arising from stratification. The associated necessary and sufficient condition for stability is given by Eq. (48). When this condition is satisfied, stratification is either neutral (when $\mathcal{F} \geq 0$ ) or stabilizing for one mode below the critical wavenumber $k_{\|}^{*}$ defined by Eq. (51) (when $\mathcal{F}<0$ ). Otherwise, stratification is either destabilizing for one mode below $k_{\|}^{*}$ (when $\mathcal{F} \geq 0$ ) or neutral (when $\mathcal{F}<0$, so that already one mode is firehose unstable).

The two parts of Eq. (39) together with Eq. (48) constitute the necessary and sufficient condition for all low-frequency, small-scale, linear modes in a nonrotating stratified medium to be stable at all $\boldsymbol{k}$. The nature and wave-vector range of the unstable modes appearing when at least one of the three stability conditions fails are shown in Table 2 .

\section{Appendix}

When the equilibrium distribution function of each plasma species (denoted with a subscript $s$ in Eqs. A6-A10 below) is bi-Maxwellian,

$f_{0}=\left(\frac{m}{2 \pi}\right)^{1.5} \frac{n_{0}}{T_{\perp 0} \sqrt{T_{\| 0}}} \exp \left(-\frac{m v_{\perp}^{2}}{2 T_{\perp 0}}\right) \exp \left(-\frac{m v_{\|}^{2}}{2 T_{\| 0}}\right)$,

the polytropic indices can be expressed in terms of the dimensionless function

$\mathcal{K}=1-\sqrt{\pi} z[1-\Phi(z)] \exp \left(z^{2}\right)$,

where

$z=-i \frac{\omega}{\sqrt{2} v_{\mathrm{T} \|} k_{\|}}$,

$v_{\mathrm{T} \|}=\sqrt{T_{\| 0} / m}$ is the parallel thermal speed, and $\Phi(z)$ is the complex error function.
In the limit $|z| \ll 1$,

$$
\begin{aligned}
\mathcal{K} & \simeq 1-\sqrt{\pi} z \\
& \simeq 1+i \sqrt{\frac{\pi}{2}}\left(\frac{\omega}{v_{\mathrm{T} \|} k_{\|}}\right) .
\end{aligned}
$$

In the limit $|z| \gg 1$,

$$
\begin{aligned}
\mathcal{K} & \simeq \frac{1}{2 z^{2}}-\frac{3}{\left(2 z^{2}\right)^{2}} \\
& \simeq-\left(\frac{v_{\mathrm{T} \|} k_{\|}}{\omega}\right)^{2}-3\left(\frac{v_{\mathrm{T} \|} k_{\|}}{\omega}\right)^{4}
\end{aligned}
$$

when $\Re(z)>-|\Im(z)|$ or, equivalently, $\Im(\omega)>-|\Re(\omega)|$, whereas the asymptotic behavior prevailing when $\Re(z)<$ $-|\Im(z)|$ (or, more generally, when $\Re(z)<0$ ) follows from the symmetry relation $\mathcal{K}(z)=\mathcal{K}(-z)-2 \sqrt{\pi} z \exp \left(z^{2}\right)$.

If we assign a subscript $s$ to all quantities pertaining to a given species and if we drop the subscript 0 denoting the equilibrium state to reduce the number of subscripts, we can write the expressions for the polytropic indices as

$$
\begin{aligned}
& \gamma_{\perp_{\perp}}=2\left(1-\sum_{s} \frac{P_{s \perp}}{P_{\perp}} \frac{T_{s \perp}}{T_{s \|}} \mathcal{K}_{s}\right) \\
& +\frac{\left(\sum_{s} q_{s} n_{s} \frac{T_{s \perp}}{T_{s \|}} \mathcal{K}_{s}\right)^{2}}{P_{\perp}\left(\sum_{s} \frac{q_{s}^{2} n_{s}}{T_{s \|}} \mathcal{K}_{s}\right)}+\gamma_{\perp_{\|}}^{2} \frac{C_{\perp}^{2}}{\mathcal{V}^{2}}, \\
& \gamma_{\perp_{\|}}=\frac{\mathcal{V}^{2}}{C_{\perp}^{2}}\left[\left(\sum_{s} \frac{\rho_{s}}{\rho} \frac{T_{s \perp}}{T_{s \|}} \mathcal{K}_{s}\right)\right. \\
& \left.-\left(\sum_{s} q_{s} n_{s} \frac{T_{s \perp}}{T_{s \|}} \mathcal{K}_{s}\right) \frac{\sum_{s} \frac{\rho_{s}}{\rho} \frac{q_{s}}{T_{s \|}} \mathcal{K}_{s}}{\sum_{s} \frac{q_{s}^{2} n_{s}}{T_{s \|}} \mathcal{K}_{s}}\right], \\
& \gamma_{\|_{\perp}}=1-\frac{P_{\perp}}{P_{\|}}\left(1-\gamma_{\perp_{\|}}\right), \\
& \gamma_{\|\|}=\frac{\mathcal{V}^{2}}{C_{\|}^{2}}+\frac{\omega^{2}}{C_{\|}^{2} k_{\|}^{2}},
\end{aligned}
$$

where

$\mathcal{V}^{2}=\left[\left(\sum_{s} \frac{\rho_{s}}{\rho} \frac{m_{s}}{T_{s \|}} \mathcal{K}_{s}\right)-\frac{\rho\left(\sum_{s} \frac{\rho_{s}}{\rho} \frac{q_{s}}{T_{s \|}} \mathcal{K}_{s}\right)^{2}}{\sum_{s} \frac{q_{s}^{2} n_{s}}{T_{s \|}} \mathcal{K}_{s}}\right]^{-1}$

Note that Eq. (A8) is in fact quite general, as it applies not only to bi-Maxwellians but also to arbitrary distribution functions. In addition, this relation is trivially satisfied both in isotropic-adiabatic MHD $\left(P_{\perp}=P_{\|}\right.$and $\gamma_{\perp_{\|}}=\gamma_{\| \perp}$; see Eq. 25) and in gyrotropic-double-adiabatic $\operatorname{MHD}\left(\gamma_{\perp_{\|}}=\right.$ $\gamma_{\| \perp}=1$; see Eq. 28). 
Acknowledgements. I am grateful to G. Belmont for his careful reading of the manuscript and for his valuable comments and suggestions.

Edited by: J. Büchner

Reviewed by: G. Belmont and another referee

\section{References}

Abraham-Shrauner, B.: Propagation of hydromagnetic waves through an anisotropic plasma, J. Plasma Physics, 1, 361-378, 1967.

André, N. and Ferrière, K. M.: Low-frequency waves and instabilities in stratified, gyrotropic, multicomponent plasmas: Theory and application to plasma transport in the Io torus, J. Geophys. Res., in press, 2004.

Belmont, G. and Mazelle, C.: Polytropic indices in collisionless plasmas: Theory and measurements, J. Geophys. Res., 97, 83278336, 1992.

Belmont, G. and Rezeau, L.: Finite Larmor radius effects: The twofluid approach, Annales Geophysicae, 5A, (2), 59-69, 1987.

Chang, Z. and Callen, J. D.: Unified fluid/kinetic description of plasma microinstabilities. Part I: Basic equations in a sheared slab geometry, Phys. Fluids B, 4, 1167-1181, 1992a.

Chang, Z. and Callen, J. D.: Unified fluid/kinetic description of plasma microinstabilities. Part II: Applications, Phys. Fluids B, 4, 1182-1192, $1992 b$.

Cheng, C. Z.: A kinetic-magnetohydrodynamic model for lowfrequency phenomena, J. Geophys. Res., 96, 21 159-21 171, 1991.

Cheng, C. Z. and Johnson, J. R.: A kinetic-fluid model, J. Geophys. Res., 104, 413-427, 1999.

Chew, G. F., Goldberger, M. L., and Low, F. E.: The Boltzmann equation and the one-fluid hydromagnetic equations in the absence of particle collisions, Proc. R. Soc. London, A, 236, 112118, 1956.

Ferrière, K. M. and André, N.: A mixed magnetohydrodynamickinetic theory of low-frequency waves and instabilities in homogeneous, gyrotropic plasmas, J. Geophys. Res., 107, 1349-1365, 2002.
Ferrière, K. M. and André, N.: A mixed magnetohydrodynamickinetic theory of low-frequency waves and instabilities in stratified, gyrotropic, two-component plasmas, J. Geophys. Res., 108, 1308-1328, 2003.

Ferrière, K. M., Zimmer, C., and Blanc, M.: Magnetohydrodynamic waves and gravitational/centrifugal instability in rotating systems, J. Geophys. Res., 104, 17 335-17 356, 1999.

Hammett, G. W. and Perkins, F. W.: Fluid moment models for Landau damping with application to the ion-temperature-gradient instability, Phys. Rev. Lett., 64, 3019-3022, 1990.

Hau, L.-N. and Sonnerup, B. U. Ö.: On slow-mode waves in an anisotropic plasma, Geophys. Res. Lett., 20, 1763-1766, 1993.

Kato, Y., Tajiri, M., and Taniuti, T.: Propagation of hydromagnetic waves in collisionless plasma, I, J. Phys. Soc. Japan, 21, 765777, 1966.

Krall, N. A. and Trivelpiece, A. W.: Principles of plasma physics, McGraw-Hill, New-York, 1973.

Newcomb, W. A.: Convective instability induced by gravity in a plasma with frozen-in magnetic field, Phys. Fluids, 4, 391-396, 1961.

Northrop, T. G.: The adiabatic motion of charged particles, Interscience, New-York, 1963.

Passot, T. and Sulem, P. L.: Long-Alfvén-wave trains in collisionless plasmas. II. A Landau-fluid approach, Phys. Plasmas, 10, 3906-3913, 2003.

Passot, T. and Sulem, P. L.: A fluid description for Landau damping of dispersive MHD waves, Nonlin. Proc. Geophys., 11, 245-258, 2004,

SRef-ID: 1607-7946/npg/2004-11-245.

Siscoe, G. L.: Solar system magnetohydrodynamics, SolarTerrestrial Physics, edited by: Carovillano, R. L. and Forbes, J. M., p. 11-100, Reidel Publishing Company, 1983.

Snyder, P. B., Hammett, G. W., and Dorland, W.: Landau fluid models of collisionless magnetohydrodynamics, Phys. Plasmas, 4, 3974-3985, 1997.

Tajiri, M.: Propagation of hydromagnetic waves in collisionless plasma, II, Kinetic approach, J. Phys. Soc. Japan, 22, 1482-1494, 1967.

Wang, B.-J. and Hau, L.-N.: MHD aspects of fire-hose type instabilities, J. Geophys. Res., 108, 1463-1474, 2003. 